UDC 576

LBC 22.311

\title{
STRUCTURALAND FUNCTIONAL CHARACTERISTICS OF ADAMTS-4 AND ADAMTS-5: A REVIEW
}

\author{
Anastasia A. Korchagina \\ Volgograd State University, Volgograd, Russian Federation \\ Lyudmila V. Derevshchikova \\ Volgograd State University, Volgograd, Russian Federation
}

\begin{abstract}
ADAMTS-4 and -5 are aggrecanases that are involved in the development of osteoarthrosis, one of the most common diseases at the moment, due to which a large number of people suffer annually. By some estimates, $9.6 \%$ of men and $18 \%$ of women over the age of 60 have symptomatic osteoarthrosis. This review discusses the currently known data on the structural features and enzymatic activity of these enzymes. The structures of ADAMTS-4 and -5 are similar, they contain 7 domain sites: the signal section, the N-terminal prodomain, the catalytic domain, the disintegrin-like domain, the thrombospodin motif, the cysteine-rich domain, and the spacer domain. In addition to all these elements, ADAMTS-5 has an additional thrombospodin motif at the end. ADAMTS- 4 and -5 cleaves aggrecan in 5 different binding sites. However, the Glu373-Ala374 site probably plays the most important role in the pathogenesis, since when this bond is broken, the whole aggrecan molecule loses its integrity, which leads to the destruction of cartilage and the development of the disease. In the course of the analysis of the information, the authors have found that the participation of ADAMTS- 4 and -5 in the development of osteoarthritis varies greatly depending on the type of organism. The researchers have established that in mice ADAMTS-4 plays the largest role in the destruction of aggrecan, while in humans ADAMTS- 5 or both aggrecanases influence the development of osteoarthritis. The revealed differences are not fully described; therefore, this review summarizes the already known results in this area, which will facilitate further research in this direction.
\end{abstract}

Key words: ADAMTS-4, ADAMTS-5, structure, domains, binding sites, osteoarthritis.

Citation. Korchagina A.A., Derevshchikova L.V. Structural and Functional Characteristics of ADAMTS-4 and ADAMTS-5: A Review. Natural Systems and Resources, 2020, vol. 10, no. 1, pp. 12-21. (in Russian). DOI: https:// doi.org/10.15688/nsr.jvolsu.2020.1.2

УДК 576

ББК 22.311

\section{СТРУКТУРНО-ФУНКЦИОНАЛЬНАЯ ХАРАКТЕРИСТИКА} ADAMTS-4 И ADAMTS-5: ОБ3ОР

\author{
Анастасия Александровна Корчагина \\ Волгоградский государственный университет, г. Волгоград, Российская Федерация \\ Людмила Владимировна Деревщикова \\ Волгоградский государственный университет, г. Волгоград, Российская Федерация
}

Аннотация. ADAMTS-4 и -5 - это аггреканазы, которые участвуют в процессе развития остеоартроза, одного из самых распространенных на данный момент заболеваний, из-за которого ежегодно страдает большое количество населения. По некоторым оценкам 9,6 \% мужчин и 18 \% женщин в возрасте после 60 лет имеют симптоматический остеоартроз. В данном обзоре рассматриваются известные на сегодняшний день данные о структурных особенностях и ферментативной активности данных ферментов. Структуры ADAMTS-4 и -5 схо- 
жи, они содержат 7 доменных участков: сигнальная область, $\mathrm{N}$-концевой продомен, каталитический домен, дезинтегринподобный домен, мотив тромбосподина, богатый цистеином домен и спейсерный домен. Помимо всех этих элементов, у ADAMTS-5 имеется в конце дополнительный мотив тромбосподина. ADAMTS-4 и -5 расщепляет аггрекан в 5 различных участках связывания. Однако наиболее важную роль в патогенезе, вероятно, играет участок Glu373-Ala374, поскольку при разрушении этой связи вся молекула аггрекана теряет целостность, что приводит к разрушению хряща и развитию заболевания. В ходе анализа информации было обнаружено, что участие ADAMTS-4 и ADAMTS-5 в развитии остеоартроза сильно разнится в зависимости от вида организма. Выяснено, что у мышей наибольшую роль в разрушении аггрекана играет ADAMTS-4, в то время как у человека ADAMTS-5 или обе аггреканазы влияют на развитие остеоартроза. Выявленные различия до конца не описаны, поэтому данный обзор обобщает уже известные результаты в этой области, что будет способствовать дальнейшему проведению исследования в этом направлении.

Ключевые слова: ADAMTS-4, ADAMTS-5, структура, домены, участки связывания, остеоартроз.

Цитирование. Корчагина А. А., Деревщикова Л. В. Структурно-функциональная характеристика ADAMTS-4 и ADAMTS-5: обзор // Природные системы и ресурсы. - 2020. - T. 10, № 1. - С. 12-21. - DOI: https://doi.org/10.15688/nsr.jvolsu.2020.1.2

Введение. ADAMTS-4 и -5 являются аггреканазами суставного хряща. Они представляют собой внеклеточные протеолитические ферменты, которые являются членами семейства ADAMTS (дезинтегрин и металлопротеиназа с мотивами тромбоспондина). Аггреканазы действуют на аггреканы - хрящевые специфичные протеогликановые ядерные белки, которые обеспечивают хрящу сжимаемость и эластичность путем набухания и увлажнения коллагеновой сети [42]. Потеря аггрекана считается критическим ранним событием при остеоартрозе (ОА), происходящим первоначально на поверхности сустава и прогрессирующим в более глубокие зоны. За этим следует деградация коллагеновых фибрилл и механическое разрушение ткани [9]. Аггреканаза-1 (ADAMTS-4) и аггреканаза-2 (ADAMTS-5) расщепляют аггрекан на уникальном сайте, названном «сайтом аггреканазы»-Glu373-Ala374 [25; 26].

Структура белков ADAMTS-4 и ADAMTS-5. Белки ADAMTS представляют собой семейство цинк-зависимых ферментов в металлопротеиназах семейства метцинцин $[15 ; 20 ; 35 ; 37]$. Они состоят из сигнальной области (Signalsequence, SS), N-концевого продомена (Pro), каталитического домена
(Catalytic, Cat), дезинтегринподобного домена (Disintegrinlike, Dis), одного или нескольких мотивов тромбоспондина (TS), богатого цистеином домена (Cysteinrich, CysR) и спейсерного домена (Spacer, Sp) [12; 36]. Структура белков ADAMTS-4 и ADAMTS-5 схожа и отличается только одним доменом: у ADAMTS-5 есть дополнительный второй мотив тромбоспондина, следующий за спейсерным [4] (рис. 1).

Сигнальная последовательность - короткая аминокислотная последовательность в составе белка, которая обеспечивает котрансляционный или посттрансляционный транспорт белка в соответствующую органеллу. После доставки белка в органеллу сигнальный пептид может отщепляться под действием специфической сигнальной протеазы.

Домены имеют различные консервативные последовательности, которые играют ту или иную важную роль, которые известны на данный момент (см. рис. 2). Доменные участки, играющие важную роль в разрушении аггрекана, представлены в таблице.

Продомен содержит три консервативных остатка Cys, обнаруженных в других ADAMTS $[18 ; 34]$. Первые два Суs находятся в консервативных последовательностях

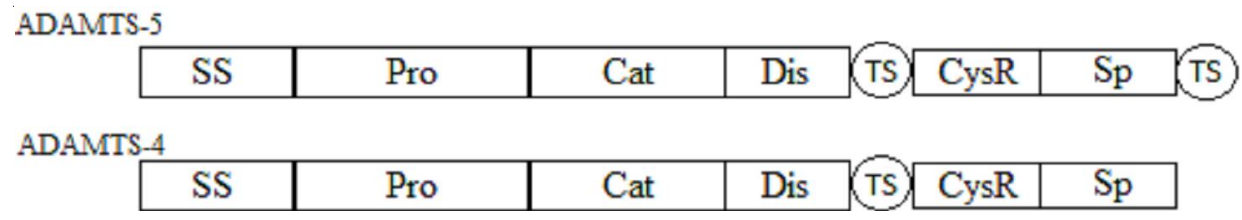

Рис. 1. Структура белков ADAMTS-4 и -5 


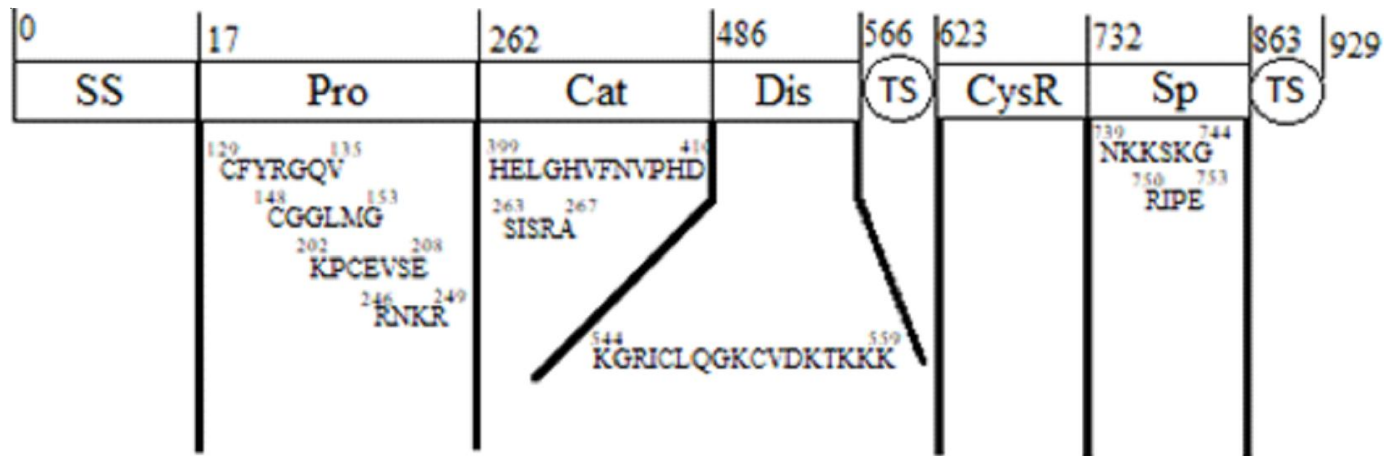

Рис. 2. Консервативные последовательности и участки доменов на примере ADAMTS-5

Краткая характеристика доменов ADAMTS-4 и ADAMTS-5

\begin{tabular}{|c|c|c|c|}
\hline Наименование домена & Сокращение & $\begin{array}{c}\text { Длина аминокислотной } \\
\text { последовательности }\end{array}$ & У частки доменов * \\
\hline Сигнальная область & SS & $0-17$ & - \\
\hline \multirow[t]{4}{*}{ Продомен } & \multirow[t]{4}{*}{ Pro } & \multirow[t]{4}{*}{$18-262$} & CFYRGQV (129-135) \\
\hline & & & CGGLMG (148-153) \\
\hline & & & KPCEVSE (202-208) \\
\hline & & & RNKR (246-249) \\
\hline Каталитический домен & Cat & $263-486$ & HELGHVFNVPHD (399-410) \\
\hline Дезинтегринподобный домен & Dis & $487-566$ & KGRICLQGKCVDKTKKK (544-559) \\
\hline Мотив тромбоспондина & $\mathrm{TS}^{1}, \mathrm{TS}^{2} * *$ & $\begin{array}{l}566-623, \\
864-929 * *\end{array}$ & - \\
\hline Богатый цистеином домен & CysR & $624-732$ & - \\
\hline Спейсерный домен & Sp & $733-863$ & NKKSKG (739-744) \\
\hline
\end{tabular}

Примечания. * - участки доменов, связанные с влиянием данных белков на хондроциты, изученные на момент написания работы; ** - второй TS² находится после спейсерного домена у ADAMTS-5.

CFYRGQV (позиции 129-135) и CGGLMG (позиции 148-153). Третий остаток Суs расположен близко к концу продоменов (позиция 202) и находится в последовательности (KPCEVSE). Пропептид заканчивается в основной области RNKR (позиции 246-249), которая может соответствовать последовательности узнавания пропротеин-конвертазы для генерации зрелого фермента.

Каталитический домен включает последовательность HELGHVFNVPHD (позиции 399-410), участвующую в координации каталитического атома цинка в активном центре металлопротеиназ. Этот мотив заканчивается остатком Asp, который отличает ADAM и ADAMTS от других металлопротеиназ, таких как MMP, которые содержат консервативный остаток Ser в этом положении.

Несколько остатков С-конца этого сайта, есть остаток Met (позиция 427), который формирует характеристику «поворот поворота Met» для ADAM, ADAMTS и MMP. Каталитический домен также содержит восемь консервативных остатков Суs, присутствующих в соответствующей области всех ADAMTS. А еще в него входит участок N-терминального неоэпитепа, который вызывает иммунную реакцию на рак и располагается в консервативном участке SISRA (263-267).

Каталитические домены протеиназ ADAMTS имеют высокую степень сходства и содержат цинк-связывающую последовательность, в которой каталитический цинк координируется тремя остатками гистидина. Такое расположение облегчается за счет консервативного глицина, который обеспечивает плотную петлю шпильки и позволяет третьему гистидину занимать свое правильное положение $[13 ; 21]$. Как и во всех ММР и адамализинах, за цинк-связывающей последовательностью на коротком расстоянии С-конца следует консервативный остаток метионина, расположение активного сайта, которое было названо «метцинцин-типа». Этот метионин представляет собой «met-turn», крутой поворот, выполненный в виде правого винта, кото- 
рый, по-видимому, выполняет важную функцию в структуре активного центра [40].

За каталитическим доменом следует область с 25-45 \% идентичностью с дезинтегринами яда змеи, хотя он не содержит цистеинового расположения последних [30]. В связи с этим указанный домен был назван дезинтегриноподобным, хотя в настоящее время нет опубликованных доказательств того, что этот домен ADAMTS взаимодействует с интегринами.

B отличие от белков ADAM, протеиназы ADAMTS обладают хорошо консервативным повтором, подобным тромбоспондину типа 1, гомологичному повторам типа I тромбоспондинов 1 и 2 [33], между дисинтегринподобным и богатым цистеином доменом. По аналогии с тромбоспондинами 1 и 2 [2] центральный TS протеиназ ADAMTS, как полагают, функционирует как сульфатированный гликозаминогликан-связывающий домен. Смежный гиалуронан-связывающий мотив находится в последовательности KGRICLQGKCVDKTKKK (позиции 544-559).

CysR является хорошо консервативной последовательностью, богатой цистеином, содержащей 10 остатков цистеина. В отличие от белков ADAM, в которых за CysR следуют повторы, подобные эпидермальному фактору роста (EGF), трансмембранный домен и $\mathrm{C}$-концевая цитозольная область, все протеиназы ADAMTS обладают взамен свободной от цистеина спейсерной области. Этот домен варьируется по длине и содержит несколько консервативных гидрофобных остатков в $\mathrm{N}$-концевой части и чрезвычайно вариабельную С-концевую часть. Он содержит гепарин связывающий мотив, который находится в консервативной последовательности NKKSKG (739-744). Также в спейсерный домен входит автолитический C-терминальный неоэпитоп, который располагается в последовательности RIPE (750-753) и распознается иммунной системой как мишени для Т-клеток и может вызывать иммунный ответ на рак.

Экспрессия различных домен-делеционных конструкций мышиного ADAMTS-1 выявила последовательность спейсера CysR в качестве домена, связывающего функциональный внеклеточный матрикс (ЕСM) [14]. Эта роль была подтверждена исследовани- ем обработанных на С-конце форм человеческого ADAMTS-4, которые в сочетании с конструкцией делеции, не имеющей последовательности CysR-спейсера, показали, что эти домены также связываются как с гепарином, так и с гликозаминогликанамиаггрекана (преимущественно кератана и хондроитинсульфаты) [13].

Три предполагаемые гепарин-связывающие последовательности были идентифицированы в последовательности спейсера CysR ADAMTS-4, одна в CysR и две в спейсере, и было показано, что пептиды, соответствующие этим последовательностям, ингибируют связывание ADAMTS-4 с гепарином [13]. Спейсерный домен и домены TS типа 1 важны для тесного взаимодействия с внеклеточным матриксом. Консервативный цистеин, присутствующий в мотиве переключения цистеина, связывает каталитический ион цинка, таким образом ингибируя фермент. Диссоциация цистеина от иона цинка при высвобождении активирующего пептида активирует фермент [21].

Особенности ферментативной активности. Эти ферменты помимо структуры отличаются друг от друга ферментативными способностями. Fushimi и соавторы использовали химерные ферменты, в которых каталитические домены ADAMTS-4 и -5 были заменены друг с другом. Они обнаружили, что ферментативная активность ADMTS-5 в четыре раза больше по сравнению с ADAMTS-4 в регионе IGD (межглобулярный домен аггрекана) и в 2,5 раза в области аггрекана CS-2 (хондроитин сульфат-2, домен аггрекана) [16].

Также сообщалось, что расщепление ADAMTS-4, но не ADAMTS-5, на IGD (межглобулярный домен) повлиял возраст животных и человека, которое вероятно связано с различиями в замещении кератансульфата в этом домене [32]. Также это расщепление может быть связано со спаттерн гликозилированием аггрекана, который меняется с возрастом [24; 27]. Более того, было показано, что ADAMTS-4 может расщеплять другие протеогликаныхондроитинсульфата, в том числе бревикан и версикан после Glu393 и Glu441 соответственно [5; 19]. ADAMTS-4 также расщепляет олигомерный хрящ матриксный белок (СОМР), а также фибромодулин и деко- 
рин, указывая на то, что протеолитический спектр этой подгруппы протеиназы не ограничиваются расщеплением только протеогликанов как и в случае ADAMTS-5 [9; 23; 41].

Расщепление аггрекана ADAMTS-4 и -5 происходит в связи Glu373-Ala374, а также в четырех других местах (рис. 3). Исследования invitro показали, что расщепление в этих четырех местах на самом деле более эффективно, чем расщепление в связи Glu373-Ala374 [23]. Тем не менее расщепление в связи Glu373-Ala374, вероятно, более важно в патогенезе остеоартроза поскольку потеря целостности в связи с этим приводит к потере всей молекулы аггрекана, что очень вредно для целостности и функции хряща [14]. Действительно, расщепление аггрекана по связи Glu373-Ala374 считается надежным маркером активности артроза [20; 29] и фрагментов, полученных в результате этого специфического расщепления, был идентифицирован в синовиальной жидкости пациентов с ОА [39]. Кроме того, генетически модифицированные мыши со связью Glu373-Ala374, которая устойчива к расщеплению, характеризуются уменьшенной потерей аггрекана и эрозией хряща на моделях остеоартроза [25]. Интересно, что у мышей, нокаутированных по ADAMTS-5, аггреканолиз все еще происходит в сайтах, отличных от сайта Glu373-Ala374, что позволя- ет предположить, что он может быть связан c ADAMTS-4 или некоторой другой аггреканазой [3].

Аггрекан имеет два сайта расщепления, расположенные в домене GG / G2 IGD. Asn341Phe342 является основным сайтом расщепления MMP, тогда как Glu373-Ala374 расщепляется аггреканазой. Есть также четыре сайта расщепления в области аггрекана, богатой сульфатом хондроитина, которая, как известно, расщепляется, по крайней мере, ADAMTS-4 и ADAMTS-5. Черные стрелки на диаграмме обозначают место, где происходит расщепление [36].

Хотя было показано, что ADAMTS-4 эффективно расщепляет аггрекан invitro, ненокаутированные мыши ADAMTS-4 не защищены от потери аггрекана. Однако нокаутные мыши ADAMTS-5 защищены от разрушения суставов, и, таким образом, в настоящее время считается, что ADAMTS-5 является основным медиатором разрушения хряща при артрозе, по крайней мере у мышей $[1 ; 10]$. Это подтверждается наблюдением, что IL-1б (Интерлейкин 1 альфа, ингибитор ADAMTS) сильно стимулирует экспрессию ADAMTS-5, но не ADAMTS-4 у мышей [1].

Хотя ADAMTS-5 (а не ADAMTS-4) может играть важную роль в развитии артроза на мышиной модели, исследования с участи-
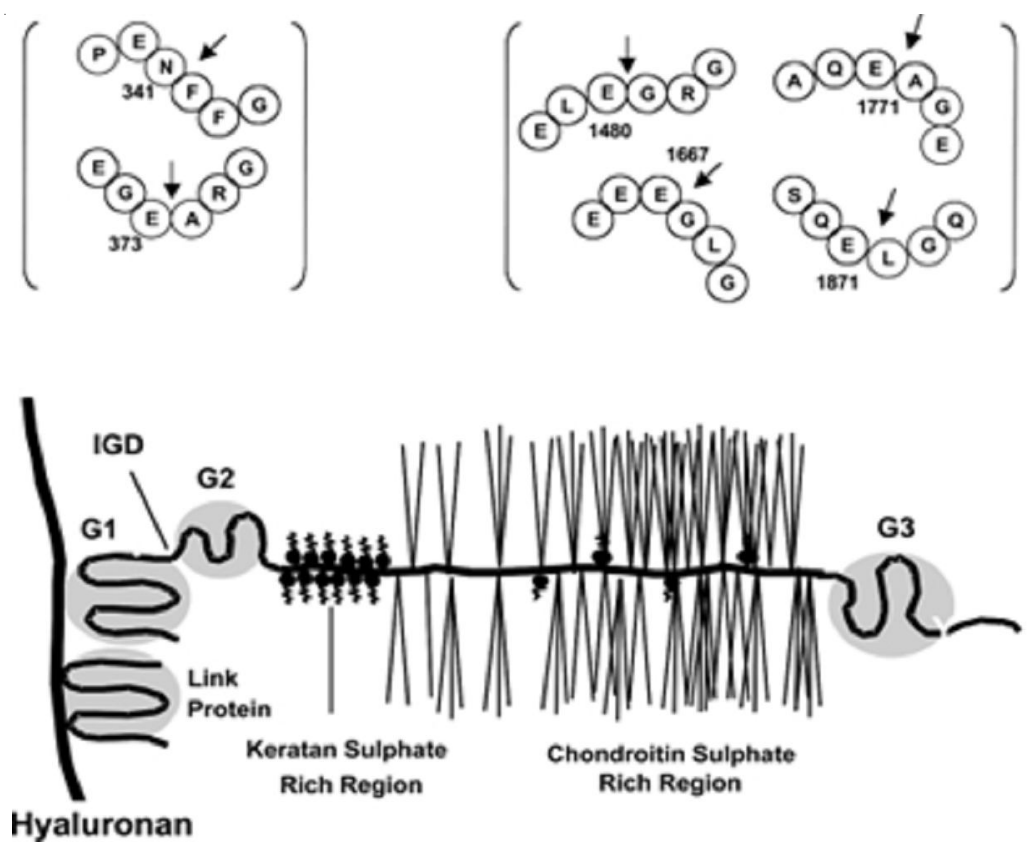

Рис. 3. Схематическое изображение аггрекана и его известных сайтов расщепления 
ем человеческой ткани, кажется, предполагают обратное - ADAMTS-4 (а не ADAMTS-5) является основным молекулярным игроком в артрозе человека. Например, генетическая изменчивость ADAMTS-5 у человека, по-видимому, не влияет на восприимчивость к ОА [17]. Кроме того, лечение онкостатином М и IL-1 $\beta$ или TNF- $\alpha$ в хондроцитах или эксплантатах хряща человека приводит к выраженной индукции ADAMTS-4 с меньшей активацией ADAMTS-5 [7]. Этот процесс, по-видимому, опосредован передачей сигналов Ras [6]. Кроме того, некоторые данные показывают, что только уровни ADAMTS-4 повышаются в ОА хряще $[11 ; 22 ; 28]$. Однако обработка эксплантированной человеческой ткани IL-1 $\alpha$ предполагает, что и ADAMTS-4, и -5 важны при заболевании человека [7]. ADAMTS-4 и -5 высоко экспрессируются в ОА хряще человека, и обе молекулы, по-видимому, способствуют потере аггрекана как в нормальном, так и в ОА хряще [8; 31; 38]. Таким образом, хотя ADAMTS-5 (а не ADAMTS-4) важен для мышей, именно ADAMTS-4 или, возможно, оба ADAMTS-4 и -5 играют ключевую роль при артрозе человека.

Заключение. На сегодняшний день изучены все доменные участки ADAMTS-4 и -5, их структурное расположение и функциональные характеристики. Также известны сайты связывания, из которых наибольшую роль в патогенезе играет участок Glu373-Ala374. Ферментативная активность ADAMTS-4 и ADAMTS-5 отличается у разных организмов. У человека наибольшую роль в развитии ОА играет ADAMTS-4, у мышей - ADAMTS-5. Проведение экспериментальных исследований для поиска возможных путей решения проблем предотвращения и лечения данного заболевания в таком случае на мышах и перенос результатов на человека может быть неэффективным. Необходимо более детально изучить этот вопрос и определить причину данных различий.

\section{СПИСОК ЛИТЕРАТУРЫ}

1. A Disintegrin and Metalloproteinase with Thrombospondin Motifs-5 (ADAMTS-5) Rormscatalytically Active Oligomers / H. J. Kosasih [et al.] // J Biol Chem. - 2016. - Vol. 291, № 7. - P. 31973208. - DOI: https://doi.org/10.1074/jbc.M115.704817.
2. ADAMTS4 Cleaves at the Aggrecanase Site (Glu373-Ala374) and Secondarily at the Matrix Metalloproteinase Site (Asn341-Phe342) in the Aggrecan Interglobular Domain / J. Westling [et al.] // J Biol Chem. - 2002. - Vol. 277, № 18. - P. 16059 16066. - DOI: https://doi.org/10.1074/jbc.M108607200.

3. ADAMTS-5 Deficiency Does not Block Aggrecanolysis at Preferred Cleavage Sites in the Chondroitin Sulfate-Rich Region of Aggrecan / C. J. East [et al.] // J Biol Chem. - 2007. - Vol. 282, № 12. -P. 86328640. - DOI: https://doi.org/10.1074/jbc.M605750200.

4. ADAMTS-5: the Story so Far / A. J. Fosang [et al.] // Eur Cell Mater. - 2008 - Vol. 15. - P. 11-26. DOI: https://doi.org/10.22203/eCM.v015a02.

5. ADAMTS-7: a Metalloproteinase that Directly Binds to and Degrades Cartilage Oligomeric Matrix Protein / C. J. Liu [et al.] // FASEB J. - 2006. Vol. 20, № 7. - P. 988-990. - DOI: https://dx.doi.org/ 10.1096\%2Ffj.05-3877fje.

6. Adaptor Proteins and Ras Synergistically Regulate IL-1-Induced ADAMTS-4 Expression in Human Chondrocytes / R. Ahmad [et al.] // J Immunol. 2009. - Vol. 182, № 8. - P. 5081-5087. - DOI: https:// doi.org/10.4049/jimmunol.0803544.

7. Aggrecan Degradation in Human Articular Cartilage Explants is Mediated by Both ADAMTS-4 and ADAMTS-5. / R. H. Song [et al.] // Arthritis Rheum.2007. - Vol. 56, № 2. -P. 575-585. - DOI: https://doi.org/ $10.1002 /$ art.22334.

8. Aggrecanolysis in Human Osteoarthritis: Confocal localization and Biochemical Characterization of ADAMTS5-Hyaluronan Complexes in Articular Cartilages / A. Plaas [et al.] // Osteoarthritis Cartilage. 2007. - Vol. 15, № 7. - P. 719-734. - DOI: https://doi.org/ 10.1016/j.joca.2006.12.008.

9. Apte, S. S. A Disintegrin-Like and Metalloprotease (Reprolysin-Type) with Thrombospondin Type 1 Motif (ADAMTS) Superfamily: Functions and Mechanisms / S. S. Apte // J Biol Chem. - 2009. - Vol. 284, № 46. - P. 31493 31497. - DOI: https://doi.org/10.1074/jbc.R109.052340.

10. Apte, S. S. Anti-ADAMTS5 Monoclonal Antibodies: Implications for Aggrecanase Inhibition in Osteoarthritis / S. S. Apte// Biochem J. -2016. - Vol. 473, №1.-P.e1-e4.-DOI:https://doi.org/10.1042/BJ20151072.

11. Association Between the Abnormal Expression of Matrix-Degrading Enzymes by Human Osteoarthritic Chondrocytes and Demethylation of Specific CpG Sites in the Promoter Regions // H. I. Roach [et al.] // Arthritis Rheum. - 2005. - Vol. 52, № 10. P. 3110-3124. - DOI: https://doi.org/10.1002/art.21300.

12. Association of a nsSNP in ADAMTS14 to Some Osteoarthritis Phenotypes / J. Rodriguez-Lopez [et al.] // Osteoarthritis and Cartilage. - 2009. - Vol. 17, № 3. - P. 321-327. - DOI: https://doi.org/10.1016/ j.joca.2008.07.012. 
13. Autocatalytic Cleavage of ADAMTS-4 (Aggrecanase-1) Reveals Multiple GlycosaminoglycanBinding Sites / C. R. Flannery [et al.] // J Biol. Chem. 2002. - Vol. 277, №45. - P. 42775-42780. - DOI: https:// doi.org/10.1074/jbc.M605750200.

14. Blocking Aggrecanase Cleavage in the Aggrecan Interglobular Domain Abrogates Cartilage Erosion and Promotes Cartilage Repair / C. B. Little [et al.] // J Clin Invest. - 2007. - Vol. 117, № 6. - P.1627-1636. DOI: https://dx.doi.org/10.1172\%2FJCI30765.

15. Cerdà-Costa, N. Architecture and Function of Metallopeptidase Catalytic Domains / N. CerdàCosta, F.X. Gomis-Rüth // Protein Sci. - 2014. - Vol. 23, № 2. - P. 123-144. - DOI: https://dx.doi.org/10. $1002 \% 2 F$ pro. 2400 .

16. Functional Differences of the Catalytic and Non-Catalytic Domains in Human ADAMTS-4 and ADAMTS-5 in Aggrecanolytic Activity / K. Fushimi [et al.] // J Biol Chem. - 2008. - Vol. 283, № 11. - P. 67066716. - DOI: https://doi.org/10.1074/jbc.M708647200.

17. Genetic Variation Including Nonsynonymous Polymorphisms of a Major Aggrecanase, ADAMTS-5, in Susceptibility to Osteoarthritis / J. Rodriguez-Lopez [et al.] // Arthritis Rheum. - 2008. - Vol. 58, № 2. P. 435-441. - DOI: https://doi.org/10.1002/art.23201.

18. Gomis-Rüth, F. X. Catalytic Domain Architecture of Metzincin Metalloproteases / F. X. Gomis-Rüth // J Biol Chem. - 2009. - Vol. 284, № 23. - P. 15353-15357. - DOI: https://doi.org/10.1074/ jbc.R800069200.

19. Gottschall, P. E. ADAMTS Expression and Function in Central Nervous System Injury and Disorders / P. E. Gottschall, M. D. Howell // Matrix Biology. - 2015. - Vol. 44, № 46. - P. 70-76. - DOI: https://doi.org/10.1016/j.matbio.2015.01.014.

20. Hooper, N. M. Families of Zinc Metalloproteases / N. M. Hooper // FEBS Lett. - 1994. Vol. 354, № 1. - P. 1-6. - DOI: https://doi.org/10.1016/ 0014-5793(94)01079-X.

21. Human Ehlers-Danlos Syndrome Type VII C and Bovine Dermatosparaxis are Caused by Mutations in the Procollagen I N-Proteinase Gene / A. Colige [et al.] // Am. J. Hum. Genet. - 1999. - Vol. 65. - P. 308317. - DOI: https://doi.org/10.1086/302504.

22. Hyaluronan Inhibits Expression of ADAMTS4 (Aggrecanase-1) in Human Osteoarthritic Chondrocytes / T. Yatabe [et al.] // Ann Rheum Dis. 2009. - Vol. 68, № 6. - P. 1051-1058. - DOI: https:// dx.doi.org/10.1136\%2Fard.2007.086884.

23. Identification of Aggrecanase Activity in Medium of Cartilage Culture / K. Sugimoto [et al.] // J. Biochem. - 1999. - Vol. 126. - P. 449-455. - DOI: https://dx.doi.org/10.1136\%2Fard.2007.086884.

24. Induction of Aggrecanase 1 (ADAM-TS4) by Interleukin-1 Occurs Through Activation of Constitutively Produced Protein / M. A. Pratta [et al.]
// Arthritis Rheum. - 2003. - Vol. 48, № 1. - P. 119133. -DOI: https://doi.org/10.1002/art.10726.

25. Inhibition of ADAM-TS4 and ADAM-TS5 Prevents Aggrecan Degradation in Osteoarthritic Cartilage/ A. M. Malfait [et al.] // J Biol Chem. - 2002. Vol. 277, № 25. - P. 22201-22208. - DOI: https://doi.org/ 10.1074/jbc.M200431200.

26. Lin, E. A. The Role of ADAMTSs in Arthritis /E. A. Lin, C. J. Liu// Protein \& Cell. - 2010. - Vol. 1, № 1. P. 33-47.-DOI: https://doi.org/10.1053/joca. 2001.0427.

27. MMPs are Less Efficient than ADAMTS5 in Cleaving Aggrecan Core Protein / M. Durigova [et al.] // Matrix Biol.-2011. - Vol. 30, № 2. -P. 145-153. -DOI: https://dx.doi.org/10.1016\%2Fj.matbio.2010.10.007.

28. Nagase, H. Aggrecanases and Cartilage Matrix Degradation / H. Nagase, M. Kashiwagi // Arthritis Res Ther. - 2005. - Vol. 5, № 2. - P. 94-103. - DOI: https:// dx.doi.org/10.1186\%2Far630.

29. Proteolytic Activities of Human ADAMTS-5: Comparative Studies with ADAMTS-4 / C. Gendron [et al.] // J Biol Chem. - 2007. - Vol. 282, № 25. - P. 18294 18306. - DOI: https://doi.org/10.1074/jbc.m701523200.

30. Purification and Cloning of Aggrecanase-1: A Member of the ADAMTS Family of Proteins / M. D. Tortorella [et al.] // Science. - 1999. - Vol. 284, № 5420. - P. 1664-1666. - DOI: https://doi.org/10.1126/ science.284.5420.1664.

31. Relative Messenger RNA Expression Profiling of Collagenases and Aggrecanases in Human Articular Chondrocytes in vivo and in vitro. / B. Bau [et al.] // Arthritis Rheum. - 2002. - Vol. 46, № 10. P. 2648-2657. - DOI: https://doi.org/10.1002/art.10531.

32. Roughley, P. J. The Role of Aggrecan in Normal and Osteoarthritic Cartilage / P. J. Roughley, J. S. Mort // Journal of Experimental Orthopaedics. 2014. - Vol. 1, № 8. - P. 1-11. - DOI: https://doi.org/ 10.1186/s40634-014-0008-7.

33. Structural and Inhibition Analysis Reveals the Mechanism of Selectivity of a Series of Aggrecanase Inhibitors / M. D. Tortorella [et al.] // The Journal of Biological Chemistry. - 2009. - Vol. 284, № 36. P. 24185-24191. - DOI: https://dx.doi.org/10.1074\% 2Fjbc.M109.029116.

34. Tang, B. L. ADAMTS: a Novel Family of Extracellular Matrix Proteases / B. L. Tang // The International Journal of Biochemistry \& Cell Biology.2019. - Vol. 33, № 1. - P. 33-44. - DOI: https://doi.org/ 10.1016/s1357-2725(00)00061-3.

35. Tang, B.L. ADAMTS: a Novel Family of Proteases with an ADAM Protease Domain and Thrombospondin 1 Repeats / B. L. Tang, W. Hong // FEBS Lett. - 1999. - Vol. 445, №2-3. -P. 223-225. - DOI: https:// doi.org/10.1016/s0014-5793(99)00119-2.

36. The ADAMTS Metalloproteinases / S. Porter [et al.] // Biochem J. - 2005. - Vol. 386, № 1. - P. 15-27. DOI: https://dx.doi.org/10.1042\%2FBJ20040424. 
37. The Metzincins: A Superfamily of Structurally Related Metalloproteinases / W. Bode [et al.] // Zoology. - 1996. - Vol. 99, № 4. - P. 237-246. - DOI: https://doi.org/10.1026/jzo.6182.

38. The Role of ADAM-TS4 (Aggrecanase-1) and ADAM-TS5 (Aggrecanase-2) in a Model of Cartilage Degradation / M. D. Tortorella [et al.] // Osteoarthritis Cartilage.-2001.-Vol.9, № 6.-P. 539-552.-DOI: https:// doi/org/10.1053/joca.2001.0427.

39. The Structure of Aggrecan Fragments in Human Synovial Fluid. Evidence for the Involvement in Osteoarthritis of a Novel Proteinase Which Cleaves the Glu373-Ala374 Bond of the Interglobular Domain / J. D. Sandy [et al.] // J Clin Invest. - 1992. - Vol. 89, № 5. - P. 1512-1516. - DOI: https://dx.doi.org/ 10.1172\%2FJCI115742.

40. Transgenic Mice with Inactive Alleles for Procollagen N-Proteinase (ADAMTS-2) Develop Fragile Skin and Male Sterility / S. W. Li [et al.] // Biochem J. - 2001 - Vol. 355. - P. 271-278. - DOI: https://dx.doi.org/10.1042\%2F0264-6021\%3A3550271.

41. Verma, P. ADAMTS-4 and ADAMTS-5: Key Enzymes in Osteoarthritis. / P. Verma, K. Dalal // J Cell Biochem. - 2011. - Vol. 112, № 12. - P. 3507-3514. DOI: https://doi.org/10.1002/jcb.23298.

42. Vertel, B. M. Aggrecan / B. M. Vertel, A. Ratcliffe // Proteoglycans: Structure, Biology and Molecular Interactions. - 2000. - P. 343-377. - DOI: https://doi.org/10.1002/jcb.23298.

\section{REFERENCES}

1. Kosasih H.J., Last K., Rogerson F.M. et al. A Disintegrin and Metalloproteinase with Thrombospondin Motifs-5 (ADAMTS-5) Forms Catalytically Active Oligomers. J Biol Chem, 2016, vol. 291, no. 7, pp. 3197-3208. DOI: https://doi.org/ 10.1074/jbc.M1 15.704817.

2. Westling J., Fosang A.J., Last K. et al. ADAMTS4 Cleaves at the Aggrecanase Site (Glu373Ala374) and Secondarily at the Matrix Metalloproteinase Site (Asn341-Phe342) in the Aggrecan Interglobular Domain. J Biol Chem, 2002, vol. 277, no. 18, pp. 16059-16066. DOI: https://doi.org/ 10.1074/jbc.M108607200.

3. East C.J., Stanton H., Golub S.B. et al. ADAMTS-5 Deficiency Does Not Block Aggrecanolysis at Preferred Cleavage Sites in the Chondroitin Sulfate-Rich Region of Aggrecan. J Biol Chem, 2007, vol. 282, no. 12, pp. 8632-8640. DOI: https:// doi.org/10.1074/jbc.M605750200.

4. Fosang A.J., Rogerson F.M., East C.J. et al. ADAMTS-5: The Story so Far. Eur Cell Mater, 2008, vol. 15, pp. 11-26. DOI: https://doi.org/10.22203/ eCM.v015a02.
5. Liu C.J., Kong W., Ilalov K. et al. ADAMTS-7: A Metalloproteinase That Directly Binds to and Degrades Cartilage Oligomeric Matrix Protein. FASEBJ, 2006, vol. 20, no. 7, pp. 988-990. DOI: https://dx.doi.org/ 10.1096\%2Ffj.05-3877fje.

6. Ahmad R., Sylvester J., Ahmad M. et al. Adaptor Proteins and Ras Synergistically Regulate IL1-Induced ADAMTS-4 Expression in Human Chondrocytes. J Immunol, 2009, vol. 182, no. 8, pp. 5081-5087. DOI: https://doi.org/10.4049/ jimmunol.0803544.

7. Song R.H., Tortorella M.D., Malfait A.M. et al. Aggrecan Degradation in Human Articular Cartilage Explants is Mediated by Both ADAMTS-4 and ADAMTS-5. Arthritis Rheum, 2007, vol. 56, no. 2, pp. 575-585. DOI: https://doi.org/10.1002/art.22334.

8. Plaas A., Osborn B., Yoshihara Y. et al. Aggrecanolysis in Human Osteoarthritis: Confocal Localization and Biochemical Characterization of ADAMTS5-hyaluronan Complexes in Articular Cartilages. Osteoarthritis Cartilage, 2007, vol. 15, no. 7, pp. 719-734. DOI: https://doi.org/10.1016/j.joca.2006.12.008.

9. Apte S.S. A Disintegrin-Like and Metalloprotease (Reprolysin-Type) with Thrombospondin Type 1 Motif (ADAMTS) Superfamily: Functions and Mechanisms. J Biol Chem, 2009, vol. 284, no. 6, pp. 31493-31497. DOI: https:// doi.org/10.1074/jbc.R109.052340.

10. Apte S.S. Anti-ADAMTS5 Monoclonal Antibodies: Implications for Aggrecanase Inhibition in Osteoarthritis. Biochem J, 2016, vol. 473, no. 1, pp.e1e4. DOI: https://doi.org/10.1042/BJ20151072.

11. Roach H.I., Yamada N., Cheung K.S. et al. Association Between the Abnormal Expression of Matrix-Degrading Enzymes by Human Osteoarthritic Chondrocytes and Demethylation of Specific Cpg Sites in the Promoter Regions. Arthritis Rheum, 2005, vol. 52, no. 10, pp. 3110-3124. DOI: https://doi.org/10.1002/ art.21300.

12. Rodriguez-Lopez J., Pombo-Suarez M., Loughlin J. et al. Association of a nsSNP in ADAMTS14 to Some Osteoarthritis Phenotypes. Osteoarthritis and Cartilage, 2009, vol. 17, no. 3, pp. 321-327. DOI: https:// doi.org/10.1016/j.joca.2008.07.012.

13. Flannery C.R, Zeng W., Corcoran C. et al. Autocatalytic Cleavage of ADAMTS-4 (Aggrecanase-1) Reveals Multiple Glycosaminoglycan-Binding Sites. J Biol. Chem, 2002, vol. 277, no. 45, pp. 42775-42780. DOI: https://doi.org/10.1074/jbc.M605750200.

14. Little C.B., Meeker C.T., Golub S.B. Blocking Aggrecanase Cleavage in the Aggrecan Interglobular Domain Abrogates Cartilage Erosion and Promotes Cartilage Repair. JClin Invest, 2007, vol. 117, no. 6, pp. 16271636. DOI: https://dx.doi.org/10.1172\%2FJCI30765.

15. Cerdà-Costa N., Gomis-Rüth F.X. Architecture and Function of Metallopeptidase Catalytic Domains. 
Protein Sci, 2014, vol. 23, no. 2, pp. 123-144. DOI: https://dx.doi.org/10.1002\%2Fpro.2400.

16. Fushimi K., Troeberg L., Nakamura H. et al. Functional Differences of the Catalytic and NonCatalytic Domains in Human ADAMTS-4 and ADAMTS-5 in Aggrecanolytic Activity. J Biol Chem, 2008, vol. 283, no. 11, pp. 6706-6716. DOI: https:// doi.org/10.1074/jbc.M708647200.

17. Rodriguez-Lopez J., Mustafa Z., PomboSuarez M. et al. Genetic Variation Including Nonsynonymous Polymorphisms of a Major Aggrecanase, ADAMTS-5, in Susceptibility to Osteoarthritis. Arthritis Rheum, 2008, vol. 58, no. 2, pp. 435-441. DOI: https://doi.org/10.1002/art.23201.

18. Gomis-Rüth F.X. Catalytic Domain Architecture of Metzincin Metalloproteases. J Biol Chem, 2009, vol. 284, no. 23, pp. 15353-15357. DOI: https://doi.org/10.1074/jbc.R800069200.

19. Gottschall P.E., Howell M.D. ADAMTS Expression and Function in Central Nervous System Injury and Disorders. Matrix Biology, 2015, vol. 44, no. 46, pp. 70-76. DOI: https://doi.org/10.1016/ j.matbio.2015.01.014.

20. Hooper N.M. Families ofZinc Metalloproteases. FEBS Lett, 1994, vol. 354, no. 1, pp. 1-6. DOI: https:// doi.org/10.1016/0014-5793(94)01079-X.

21. Colige A., Sieron A.L., Li S.W. et al. Human Ehlers-Danlos Syndrome Type VII C and Bovine Dermatosparaxis Are Caused by Mutations in the Procollagen I N-Proteinase Gene. Am. J. Hum. Genet, 1999, vol. 65, pp. 308-317. DOI: https://doi.org/10.1086/ 302504

22. Yatabe T., Mochizuki S., Takizawa M. et al. Hyaluronan Inhibits Expression of ADAMTS4 (Aggrecanase-1) in Human Osteoarthritic Chondrocytes. Ann Rheum Dis, 2009, vol. 68, no. 6, pp. 1051-1058. DOI: https://dx.doi.org/10.1136\%2Fard.2007.086884.

23. Sugimoto K., Takahashi M., Yamamoto Y. et al. Identification of Aggrecanaseactivity in Medium of Cartilage Culture. J. Biochem, 1999, vol. 126, pp. 449455. DOI: https://dx.doi.org/10.1136\%2Fard.2007. 086884 .

24. Pratta M.A., Scherle P.A., Yang G. et al. Induction of Aggrecanase 1 (ADAM-TS4) by Interleukin-1 Occurs Through Activation of Constitutively Produced Protein. Arthritis Rheum, 2003, vol. 48, no. 1, pp. 119-133. DOI: https://doi.org/ 10.1002/art.10726.

25. Malfait A.M., Liu R.Q., Ijiri K. et al. Inhibition of ADAM-TS4 and ADAM-TS5 Prevents Aggrecan Degradation in Osteoarthritic Cartilage. J Biol Chem, 2002, vol. 277, no. 25, pp. 22201-22208. DOI: https:// doi.org/10.1074/jbc.M200431200.

26. Lin E.A., Liu C.J. The Role of ADAMTSs in Arthritis. Protein \& Cell, 2010, vol. 1, no. 1, pp. 33-47. DOI: https://doi.org/10.1053/joca.2001.0427.
27. Durigova M., Nagase H., Mort J.S. et al. MMPs are Less Efficient Than ADAMTS5 in Cleaving Aggrecan Core Protein. Matrix Biol, 2011, vol. 30, no. 2, pp. 145-153. DOI: https://dx.doi.org/10.1016\%2Fj. matbio.2010.10.007.

28. Nagase H., Kashiwagi M. Aggrecanases and Cartilage Matrix Degradation. Arthritis Res Ther, 2005, vol. 5, no. 2, pp. 94-103. DOI: https://dx.doi.org/ $10.1186 \% 2$ Far630.

29. Gendron C., Kashiwagi M., Lim N.H. et al. Proteolytic Activities of Human ADAMTS-5: Comparative Studies with ADAMTS-4. J Biol Chem, 2007, vol. 282, no. 25, pp. 18294-18306. DOI: https:// doi.org/10.1074/jbc.m701523200.

30. Tortorella M.D., Burn T.C., Pratta M.A. Purification and Cloning of Aggrecanase-1: A Member of the ADAMTS Family of Proteins. Science, 1999, vol. 284, no. 5420, pp. 1664-1666. DOI:https://doi.org/ 10.1126/science.284.5420.1664.

31. Bau B., Gebhard P.M., Haag J. et al. Relative Messenger RNA Expression Profiling of Collagenases and Aggrecanases in Human Articular Chondrocytes in vivo and in vitro. Arthritis Rheum, 2002, vol. 46, no. 10, pp. 2648-2657. DOI: https://doi.org/10.1002/ art. 10531 .

32. Roughley P.J., Mort J.S. The Role of Aggrecan in Normal and Osteoarthritic Cartilage. Journal of Experimental Orthopaedics, 2014, vol. 1, no. 8, pp. 111. DOI: https://doi.org/10.1186/s40634-014-0008-7.

33. Tortorella M.D., Tomasselli A.G., Mathis K.J. et al. Structural and Inhibition Analysis Reveals the Mechanism of Selectivity of a Series of Aggrecanase Inhibitors. JBiol Chem, 2009, vol. 284, no. 36, pp. 2418524191. DOI: https://dx.doi.org/10.1074\%2Fjbc. M109.029116.

34. Tang B.L. Tang BLADAMTS: A Novel Family of Extracellular Matrix Proteases. The International Journal of Biochemistry \& Cell Biology, 2019, vol. 33, no. 1, pp. 33-44. DOI: https://doi.org/10.1016/s13572725(00)00061-3.

35. Tang B.L., Hong W. ADAMTS: A Novel Family of Proteases with an ADAM Protease Domain and Thrombospondin 1 Repeats. FEBS Lett, 1999, vol.4 45, no. 3, pp. 223-225. DOI: https://doi.org/ 10.1016/s0014-5793(99)00119-2.

36. Porter S., Clark I.M., Kevorkian L. et al. The ADAMTS Metalloproteinases. Biochem J, 2005, vol. 386, no. 1, pp. 15-27. DOI: https://dx.doi.org/ 10.1042\%2FBJ20040424.p

37. Bode W., Grams F., Reinemer P. et al. The Metzincins: A Superfamily of Structurally Related Metalloproteinases. Zoology, 1996, vol. 99, no. 4, pp. 237-246. DOI:https://doi.org/10.1026/jzo.6182.

38. Tortorella M.D., Malfait A.M., Deccico C. et al. The Role of ADAM-TS4 (Aggrecanase-1) and ADAMTS5 (Aggrecanase-2) in a Model of Cartilage Degradation. 
Osteoarthritis Cartilage, 2001, vol. 9, no. 6, pp. 539-552. DOI: https://doi/org/10.1053/joca.2001.0427.

39. Sandy J.D., Flannery C.R., Neame P.J. et al. The Structure of Aggrecan Fragments in Human Synovial Fluid. Evidence for the Involvement in Osteoarthritis of a Novel Proteinase Which Cleaves the Glu 373-Ala 374 Bond of the Interglobular Domain. J Clin Invest, 1992, vol. 89, no. 5, pp. 1512-1516. DOI: https://dx.doi.org/10.1172\%2FJCI1 15742.

40. Li S.W., Arita M., Fertala A. et al. Transgenic Mice with Inactive Alleles for Procollagen N-Proteinase
(ADAMTS-2) Develop Fragile Skin and Male Sterility. Biochem J, 2001, vol. 355, pp. 271-278. DOI: https:// dx.doi.org/10.1042\%2F0264-6021\%3A3550271.

41. Verma P., Dalal K. ADAMTS-4 andADAMTS-5: Key Enzymes in Osteoarthritis. J Cell Biochem, 2011, vol. 112, no. 12, pp. 3507-3514. DOI: https://doi.org/ 10.1002/jcb.23298.

42. Vertel B.M., Ratcliffe A. Aggrecan. Proteoglycans: Structure, Biology and Molecular Interactions, 2000, pp. 343-377. DOI: https://doi.org/ $10.1002 /$ jcb.23298.

\section{Information About the Authors}

Anastasia. A. Korchagina, Student, Department of Bioengineering and Bioinformatics, Volgograd State University, Prosp. Universitetsky, 100, 400062 Volgograd, Russian Federation, nastya.korchagina09@gmail.com.

Lyudmila V. Derevshchikova, Student, Department of Bioengineering and Bioinformatics, Volgograd State University, Prosp. Universitetsky, 100, 400062 Volgograd, Russian Federation, derewshikowa2017@yandex.ru.

\section{Информация об авторах}

Анастасия Александровна Корчагина, студент кафедры биоинженерии и биоинформатики, Волгоградский государственный университет, просп. Университетский, 100, 400062 г. Волгоград, Российская Федерация, nastya.korchagina09@gmail.com.

Людмила Владимировна Деревщикова, студент кафедры биоинженерии и биоинформатики, Волгоградский государственный университет, просп. Университетский, 100, 400062 г. Волгоград, Российская Федерация, derewshikowa2017@yandex.ru. 